\title{
COVID-19 outbreaks in long-term care facilities in Hong Kong
}

Tung Wai Auyeung, MD, FHKAM (Medicine)

In this issue of the Asian Journal of Gerontology and Geriatrics, The Hong Kong Geriatrics Society, represented by a group of geriatricians, describe the situation of older patients in Hong Kong. ${ }^{1}$ The article is a joint effort from a group of geriatricians working in the public sector in Hong Kong.

The article was prepared after the second wave of coronavirus disease 2019 (COVID-19), in July 2020, and at the emergence of the third wave. Because of the rapidly changing situation, some of the information and views in that article need to be updated and supplemented. The present editorial, written amid the fourth wave in mid-December 2020, serves this purpose. The authors reported that in the third wave, the first case of COVID-19 in a residential care home for the elderly (RCHE) was diagnosed on 6 July 2020; at that time, 24 residents and five healthcare workers of that RCHE were diagnosed with COVID-19. Since then, more outbreaks of COVID-19 have occurred in RCHEs. As of 9 December 2020, 31 outbreaks have been reported in RCHEs; 198 residents and 58 staff have been confirmed infected, and 740 residents have been classified as close contacts and were or are under quarantine at designated quarantine sites.

Community geriatric assessment teams (CGATs) have actively offered support during each outbreak of COVID-19 in a RCHE. The CGATs attend the RCHE usually on the same day as they are informed of the outbreak. They discuss onsite with colleagues from the Infection Control Branch of the Department of Health about the feasibility of onsite quarantine. However, all RCHEs that have had outbreak so far have been assessed as unsuitable for onsite quarantine. Residents classified as close contacts are instead relocated to designated quarantine sites, where a designated care team from the Social Welfare Department is responsible for the personal and nursing care of the residents. The transfer of these residents to quarantine sites requires coordination of multiple parties, including the Social Welfare Department staff, the quarantine site care team, the Department of Health Auxiliary Medical Service (responsible for transport), and the CGATs. The transfer usually takes place at night because of the availability of suitable transport. These transfers improve with repeated practice after multiple outbreaks of COVID-19.

In addition to the relocation of RCHE residents to quarantine sites, CGATs have also contributed to support medical care at designated quarantine sites. They are timely, usually being available on 'day zero' when the outbreak is confirmed to provide clinical information to the Social Welfare Department to decide which patients are suitable or unsuitable for care at the designated sites. Thereafter, CGATs are allocated, by a 2-month rotation, to provide professional medical and nursing support to the residents being quarantined. The responsible CGAT goes to the designated site usually on the day after the residents move in. The CGATs assess and review the health condition and medications of the patients, and provide regular onsite support. All these activities put a sizable workload on the already stressed CGATs.

In designated quarantine sites, we observed that the older people were isolated, lonely, disorientated, and sometimes delirious. They are the victims of this pandemic as many of them were infected by people either working at or visiting the RCHE. These older people have the highest mortality rate among patients with COVID-19. Despite this, when intensive care beds or ventilators are scarce, these older patients are selected out because of their premorbid conditions leading to adverse treatment outcomes.

Many people might have different reflections on this pandemic. As remarked by a senior geriatrician in the article,"When the world is focusing on the safety of the masses, the frail, the silent and the old have no voice."I hope this editorial can add a little voice speaking for them. 
I would also like to commemorate and salute to the great effort of the geriatricians and the CGAT nurses of Hong Kong who have firmly held their posts in this pandemic.

\section{REFERENCE}

1. Auyeung TW, Chan FHW, Chan TY, et al. COVID-19 and older adults: experience in Hong Kong. Asian J Gerontol Geriatr 2020;15(2):549. Crossref 